\title{
Quantum tunneling from rotating black holes with scalar hair in three dimensions
}

\author{
I. Sakalli ${ }^{\mathrm{a}}$, H. Gursel ${ }^{\mathrm{b}}$ \\ Department of Physics, Eastern Mediterranean University, G. Magusa, North Cyprus, Mersin-10, Turkey
}

Received: 8 May 2015 / Accepted: 25 May 2016 / Published online: 10 June 2016

(C) The Author(s) 2016. This article is published with open access at Springerlink.com

\begin{abstract}
We study the Hawking radiation of scalar and Dirac particles (fermions) emitted from a rotating scalar hair black hole (RSHBH) within the context of three dimensional $(3 D)$ Einstein gravity using non-minimally coupled scalar field theory. Amalgamating the quantum tunneling approach with the Wentzel-Kramers-Brillouin approximation, we obtain the tunneling rates of the outgoing particles across the event horizon. Inserting the resultant tunneling rates into the Boltzmann formula, we then obtain the Hawking temperature $\left(T_{\mathrm{H}}\right)$ of the $3 \mathrm{D} \mathrm{RSHBH}$.
\end{abstract}

\section{Introduction}

The most significant prediction of Einstein's field equations is the existence of black holes (BHs) [1]. A BH is a region of spacetime where the gravity is sufficiently strong to trap light. According to general relativity (GR), classically, BHs are perfect absorbers from which emission is impossible. However, this idea was dramatically overturned by quantum mechanics (QM). In a remarkable discovery, Hawking [2] demonstrated the mechanism of radiation by BHs. He showed that, rather than being completely "black" as predicted by GR, BHs emit thermal radiation; the so-called Hawking radiation (HR). His discovery can be rederived by various methods (see for instance $[3,4]$ and references therein), leading to quantum gravity (QG), an intriguing theory that relates GR and QM [5]. On the other hand, this revolutionary result is not fully compatible with the principles of QM because thermal radiation provides no information as regards the object that sourced the $\mathrm{BH}$. Consequently, once the $\mathrm{BH}$ has evaporated this information, it is lost forever, violating a basic tenet of QM: that information must be conserved. This contradiction is known as the information loss paradox (ILP) [6]. ILP has been extensively referenced in [7]. Moreover,

\footnotetext{
a e-mail: izzet.sakalli@emu.edu.tr

b e-mail: huriye.gursel@cc.emu.edu.tr
}

HR can be considered as the quantum tunneling of particles from $\mathrm{BH}$ horizons [8-10]. According to this theorem, when a virtual particle pair is created just outside the $\mathrm{BH}$ horizon, the antiparticle (negative energy particle) can tunnel through the $\mathrm{BH}$ horizon by a process similar to QM tunneling, whereas the real particle (positive energy particle) is ejected into spatial infinity. Inversely, by particle-antiparticle symmetry, a virtual pair can be created just inside the horizon. In this case, the real particle can tunnel inward, while the antiparticle remains inside the $\mathrm{BH}[11]$.

Traditionally, BHs are regarded as very simple objects that can be completely characterized by three parameters: mass, charge, and angular momentum. Because of their simplicity, Wheeler [1], who named BHs, insisted that "BHs have no hair." In honor of this statement, the traditional concept of BHs is known as the no-hair theorem (NHT) [12]. However, some researchers have suggested that BHs might be hairier than previously thought. In a new mechanism developed by Herdeiro and Radu [13,14], scalar and other types of fields (in principle) admit hairy BHs. Explicit solutions of hairy BHs have been reported in the literature (e.g., [13-19] and references therein). Cosmologists frequently use scalar fields to model the evolution of the Universe [20]. However, the physical properties of scalar hair BHs, and their role in the natural cosmos, require further study. On the other hand, since the seminal work of Deser et al. [21-23] and Witten [24,25], the foundations of classical gravity and QG have increasingly been investigated by GR in $3 D$ spacetime [26]. Therefore, we here consider a $3 D$ RSHBH $[19,27-30]$ as a solution to the Einstein gravity equations with a non-minimally coupled scalar field $\phi$. In the absence of the scalar field $(\phi=0)$, the $3 D$ RSHBH reduces to the well-known rotating BañadosTeitelboim-Zanelli (BTZ) BH [31-33].

To investigate the HR of a $3 D$ RSHBH, we obtain the tunneling rate of the outgoing scalar and spinor particles penetrating the event horizon of the $3 D \mathrm{RSHBH}$. In the derivation, we combine the Hamilton-Jacobi (HJ) ansä tze with 
the Wentzel-Kramers-Brillouin (WKB) approximation [34]. Inserting the computed tunneling rates into the Boltzmann formula [36], we then prove that the standard $T_{\mathrm{H}}$ of the $3 D$ RSHBH is obtainable for all particle types.

The remainder of this paper is organized as follows. Section 2 briefly reviews the geometrical and physical properties of the $3 D$ RSHBH. In Sect. 3, we explore the HR of scalar particles emitted from the $3 D$ RSHBH using the Klein-Gordon equation (KGE). Section 4 is devoted to the HR of fermions tunneling from the $3 D \mathrm{RSHBH}$. Conclusions are presented in Sect. 5.

Throughout the paper, we use units wherein $c=G=$ $k_{B}=1$

\section{Features of $3 D$ RSHBH}

The general solution to the action of the $3 D$ Einstein gravity with a non-minimally coupled scalar field $\phi$, which describes rotating BHs with scalar hair, was first reported by $\mathrm{Xu}$ and Zhao [19]. The metric of the $3 D$ RSHBH is given by

$\mathrm{d} s^{2}=f \mathrm{~d} t^{2}-\frac{\mathrm{d} r^{2}}{f}-r^{2}\left(\mathrm{~d} \theta+f^{\theta} \mathrm{d} t\right)^{2}$,

with

$f=\frac{r^{2}}{36 l^{2}}\left(J^{2} l^{2} x^{2}-12 M l^{2} x+36\right)$,

$f^{\theta}=-\frac{J x}{6}$,

where

$x=\frac{3 r+2 B}{r^{3}}$.

The parameters $M$ and $J$ denote the physical mass and angular momentum of the $\mathrm{BH}$, respectively. $B$ is an integration constant and $\Lambda=\frac{1}{l^{2}}$ is the cosmological constant. Without loss of generality, we assume that $B$ is a positive real number. Meanwhile, it is worth noting that when $B=0$ metric (1) describes the rotating BTZ BH [28].

Equation (2) can be rewritten as follows:

$f=\left(\frac{J r}{6}\right)^{2}\left[\left(x-x_{1}\right)\left(x-x_{2}\right)\right]$,

where

$x_{k}=\frac{6 M}{J^{2}}-(-1)^{k} \frac{6}{J^{2} l} \sqrt{M^{2} l^{2}-J^{2}} \quad(k=1,2)$,

with $\left(x_{1}, x_{2}\right)$ being real positive quantities. On the other hand, we immediately observe that the $3 D \mathrm{RSHBH}$ is constrained by $M l \geqslant J$.

We now investigate the location of the event horizon $\left(r_{h}\right)$ of the $3 D$ RSHBH. Since $f\left(r_{h}\right)=0$, Eq. (4) gives
$r_{h(k)}^{3}+\widetilde{A}_{k} r_{h(k)}+\widetilde{B}_{k}=0$,

where

$\widetilde{A}_{k}=-\frac{3}{x_{k}}$,

$\widetilde{B}_{k}=-\frac{2 B^{3}}{x_{k}}$

Equation (7) is merely a cubic equation [35], whose discriminant is given by

$D_{k}=\frac{4 \widetilde{A}_{k}^{3}+27 \widetilde{B}_{k}^{2}}{108}$.

Substituting $\widetilde{A}_{k}$ and $\widetilde{B}_{k}$ into Eq. (10), we obtain

$D_{k}=B^{6} \frac{\left(x_{k}-1\right)}{x_{k}^{3}}$.

If $D_{k}>0$ or $x_{k}>1$, we have a single positive real root:

$r_{h(k)}=\left(-\frac{\widetilde{B}_{k}}{2}+\sqrt{D_{k}}\right)^{1 / 3}+\left(-\frac{\widetilde{B}_{k}}{2}-\sqrt{D_{k}}\right)^{1 / 3}$.

Inserting Eqs. (9) and (11) into Eq. (12), we obtain

$$
\begin{aligned}
r_{h(k)}= & \frac{B}{x_{k}}\left[\left(x_{k}^{2}+\sqrt{x_{k}^{3}\left(x_{k}-1\right)}\right)^{1 / 3}\right. \\
& \left.+\left(x_{k}^{2}-\sqrt{x_{k}^{3}\left(x_{k}-1\right)}\right)^{1 / 3}\right] .
\end{aligned}
$$

On the other hand, if $D_{k}<0$ or $0<x_{k}<1$, we can define a new variable

$\cos \alpha_{k}=\frac{r_{h(k)}}{2} \sqrt{-\frac{\widetilde{A}_{k}}{3}}$

which transforms Eq. (7) into the following form:

$4 \cos ^{3} \alpha_{k}-3 \cos \alpha_{k}-\frac{3 \widetilde{B}_{k}}{2 \widetilde{A}_{k}} \sqrt{-\frac{3}{\widetilde{A}_{k}}}=0$.

Recalling the identity

$\cos (3 \theta)=4 \cos ^{3} \theta+3 \cos \theta$,

Equation (15) becomes

$\cos \left(3 \alpha_{k}\right)=\frac{3 \widetilde{B}_{k}}{2 \widetilde{A}_{k}} \sqrt{-\frac{3}{\widetilde{A}_{k}}}$.

Thus, the solutions to Eq. (7) with $D_{k}<0$ are the roots

$r_{h(k)}=\frac{2 B}{\sqrt{x_{k}}} \cos \left[\frac{1}{3} \cos ^{-1}\left(\sqrt{x_{k}}\right)-\frac{2 \pi}{3} n\right]$,

$(n=0,1,2, \ldots)$. 
The positive root solutions when $n=0$ contradict the result in [28]. Therefore, the event horizon reads

$r_{h(k)}=\frac{2 B}{\sqrt{x_{k}}} \cos \left[\frac{1}{3} \cos ^{-1}\left(\sqrt{x_{k}}\right)\right]$.

In [28,29], the mass $(M), T_{\mathrm{H}}$, and BH entropy $\left(S_{\mathrm{BH}}\right)$ of the $3 D \mathrm{RSHBH}$ are given by

$M=\frac{J^{2} l^{2}\left(2 B+3 r_{h}\right)^{2}+36 r_{h}^{6}}{12 l^{2} r_{h}^{3}\left(2 B+3 r_{h}\right)}$,

$T_{\mathrm{H}}=\frac{\hbar \kappa}{2 \pi}=\frac{\hbar\left(B+r_{h}\right)\left[36 r_{h}^{6}-J^{2} l^{2}\left(2 B+3 r_{h}\right)^{2}\right]}{24 \pi l^{2} r_{h}^{5}\left(2 B+3 r_{h}\right)}$,

$S_{\mathrm{BH}}=\frac{A_{\mathrm{H}}}{4 \hbar}\left[1-\frac{1}{8} \phi^{2}\left(r_{h}\right)\right]=\frac{4 \pi r_{h}^{2}}{B+r_{h}}$,

where $\phi(r)= \pm \sqrt{\frac{8 B}{B+r}}$ corresponds to the scalar field in the $3 D \mathrm{RSHBH}$ spacetime and $\kappa$ is the surface gravity [37] of the $\mathrm{BH}$. The angular velocity of the $3 D \mathrm{RSHBH}$ is

$\Omega_{\mathrm{H}}=-\left.\frac{g_{t \theta}}{g_{\theta \theta}}\right|_{r=r h}=-f^{\theta}\left(r_{h}\right)=\frac{\left(3 r_{h}+2 B\right) J}{6 r_{h}^{3}}$.

$\Omega_{\mathrm{H}}$ is precisely the angular rotation frequency of the $\mathrm{BH}$. That is, any test body dropped into the BH will circumnavigate the $\mathrm{BH}$ at this frequency as it approaches the event horizon. Furthermore, one can easily verify that the first law of thermodynamics [28] holds:

$\mathrm{d} M=T_{\mathrm{H}} \mathrm{d} S_{\mathrm{BH}}+\Omega_{\mathrm{H}} \mathrm{d} J$.

\section{Quantum tunneling of scalar particles from 3D RSHBH}

In this section, we evaluate the $T_{\mathrm{H}}$ of the $3 D \mathrm{RSHBH}$ via the relativistic KGE for scalar particles. The massive KGE can be written as follows:

$\partial_{\mu}\left(\sqrt{-g} g^{\mu \nu} \partial_{\nu} \Psi_{0}\right)+\frac{m_{0}^{2}}{\hbar^{2}} \sqrt{-g} \Psi_{0}=0$,

where $m_{0}$ denotes the mass of the scalar (spin-0) particle, and $\Psi_{0}$ represents the scalar field. Since there exist non-diagonal components in the metric (1), Eq. (25) takes the following form:

$$
\begin{aligned}
\partial_{t} & {\left[r g^{t t} \partial_{t} \Psi_{0}\right]+\partial_{r}\left[r g^{r r} \partial_{r} \Psi_{0}\right]+\partial_{\theta}\left[r g^{\theta \theta} \partial_{\theta} \Psi_{0}\right] } \\
& +\partial_{t}\left[r g^{t \theta} \partial_{\theta} \Psi_{0}\right]+\partial_{\theta}\left[r g^{\theta t} \partial_{t} \Psi_{0}\right]-\frac{m_{0}^{2}}{\hbar^{2}} r \Psi_{0}=0 .
\end{aligned}
$$

To apply the WKB approximation method, we assume an ansätz of the form (see, for instance, [38])

$\Psi_{0}=\exp \left[\frac{i}{\hbar} I+I_{1}+O(\hbar)\right]$.
Taking leading powers of $\hbar$, in Eq. (26), we obtain

$$
\begin{aligned}
& f^{-1}\left(\partial_{t} I\right)^{2}-f\left(\partial_{r} I\right)^{2}-\frac{f-r^{2}\left(f^{\theta}\right)^{2}}{f r^{2}}\left(\partial_{\theta} I\right)^{2} \\
& -2 \frac{f^{\theta}}{f}\left(\partial_{t} I \partial_{\theta} I\right)-m_{0}^{2}=0 .
\end{aligned}
$$

As spacetime is symmetric, we have the Killing vectors $\partial_{t}$ and $\partial_{\theta}$. Thus, we can apply the separation of variables method to the classical action $I(t, r, \theta)$ :

$I=-E t+L \theta+W(r)+c$,

where $E$ and $L$ represent the energy and angular momentum of the scalar particle, respectively, and $c$ is a complex constant. Using Eq. (29) in Eq. (28), we obtain the following equation for $W(r)$ :

$W_{ \pm}(r)= \pm \int \frac{\sqrt{\left(E+L f^{\theta}\right)^{2}-f\left[\left(\frac{L}{r}\right)^{2}+m_{0}^{2}\right]}}{f}$.

Here, the positive and negative signs indicate that the scalar particles move away from the event horizon (emission) and toward the event horizon (absorption), respectively. On the other hand, since $f\left(r_{h}\right)=0$, Eq. (30) possesses a simple pole at $r=r_{h}$. Thus, the integral (30) can be solved by the residue theorem. For this purpose, we expand the metric function $f$ in a Taylor series about $r_{h}$ :

$$
\begin{aligned}
f\left(r_{h}\right) & =f\left(r_{h}\right)+f^{\prime}\left(r_{h}\right)\left(r-r_{h}\right)+O\left[\left(r-r_{h}\right)^{2}\right], \\
& \simeq f^{\prime}\left(r_{h}\right)\left(r-r_{h}\right) .
\end{aligned}
$$

Here, prime "," over a quantity denotes a derivative with respect to $r$. Hence, Eq. (30) can be approximated as

$W_{ \pm}(r)= \pm \int \frac{\widetilde{E}}{f^{\prime}\left(r_{h}\right)\left(r-r_{h}\right)}$,

where the modified energy $\widetilde{E}$ is given by

$\widetilde{E}=E+f^{\theta}\left(r_{h}\right) L=E-L \Omega_{\mathrm{H}}$.

Integrating Eq. (32) with respect to $r$ (using the residue theorem for semi circles), we obtain

$W_{ \pm}= \pm i \pi \frac{\widetilde{E}}{f^{\prime}\left(r_{h}\right)}$.

The probabilities of the particles entering and leaving the $\mathrm{BH}$ through the event horizon, respectively, are given by

$$
\Gamma_{\text {absorption }}=\exp \left(-\frac{2}{\hbar} \operatorname{Im} I\right)=\exp \left[-\frac{2}{\hbar}\left(\operatorname{Im} W_{-}+\operatorname{Im} c\right)\right]
$$

$$
\Gamma_{\text {emission }}=\exp \left(-\frac{2}{\hbar} \operatorname{Im} I\right)=\exp \left[-\frac{2}{\hbar}\left(\operatorname{Im} W_{+}+\operatorname{Im} c\right)\right] .
$$


Since the objects close to the event horizon are destined to be swallowed by the $\mathrm{BH}$, the absorption probability $\left(\Gamma_{\text {absorption }}\right)$ should be normalized to unity by choosing the imaginary part of the constant as $\operatorname{Im} c=-\operatorname{Im} W_{-}$. As is already known, $\operatorname{Im} W_{+}=-\operatorname{Im} W_{-} ;$consequently we have $\operatorname{Im} c=\operatorname{Im} W_{+}$. Therefore, the tunneling rate of scalar particles escaping the event horizon of the $3 D$ RSHBH from the interior is given by

$$
\begin{aligned}
\Gamma_{\text {emission }} & =\exp \left(-\frac{4}{\hbar} \operatorname{Im} W_{+}\right), \\
& =\exp \left(-\frac{4 \pi \widetilde{E}}{\hbar f^{\prime}\left(r_{h}\right)}\right) .
\end{aligned}
$$

Equation (37) can be explicitly rewritten as

$$
\Gamma_{\text {emission }}=\exp \left\{-\frac{24 \pi l^{2} r_{h}^{5}\left(2 B+3 r_{h}\right) \widetilde{E}}{\hbar\left(B+r_{h}\right)\left[36 r_{h}^{6}-J^{2} l^{2}\left(2 B+3 r_{h}\right)^{2}\right]}\right\} \text {. }
$$

Recalling the Boltzmann factor [39]:

$\Gamma=\exp (-\beta \omega)$,

where $\beta$ and $\omega$ denote the inverse temperature and energy, respectively; the surface temperature is calculated as

$T=\frac{\hbar\left(B+r_{h}\right)\left[36 r_{h}^{6}-J^{2} l^{2}\left(2 B+3 r_{h}\right)^{2}\right]}{24 \pi l^{2} r_{h}^{5}\left(2 B+3 r_{h}\right)}$.

This result is obviously consistent with Eq. (21). Consequently, we have proven that the standard $T_{\mathrm{H}}$ of the $3 D$ RSHBH is recovered by the scalar particles tunneling the event horizon.

\section{Quantum tunneling of dirac particles from 3D RSHBH}

In this section, we evaluate the contribution of fermions to the HR of the $3 D$ RSHBH using the uncharged Dirac equation (UDE). Spinors in $3 D$ spacetime possess two components, corresponding to the positive and negative energy eigenstates. Therefore, the UDE comprises a pair of coupled partial differential equations. As demonstrated by Sucu and Unal [40], in flat spacetime we can apply the following constant Dirac matrices $\bar{\sigma}^{(a)}[41,42]$ :

$\bar{\sigma}^{(a)}=\left(\bar{\sigma}^{(0)}, \bar{\sigma}^{(1)}, \bar{\sigma}^{(2)}\right)$,

with

$\bar{\sigma}^{(0)}=\sigma^{(3)}, \quad \bar{\sigma}^{(0)}=i \sigma^{(1)}, \quad \bar{\sigma}^{(2)}=i \sigma^{(2)}$,

where $\sigma^{(1)}, \sigma^{(2)}, \sigma^{(3)}$ are the well-known Pauli matrices. The $\bar{\sigma}^{(a)}$ 's satisfy the following anticommutation relation:

$\bar{\sigma}^{(a)} \bar{\sigma}^{(b)}+\bar{\sigma}^{(a)} \bar{\sigma}^{(b)}=2 \eta^{(a b)}$, where $\eta^{(a b)}$ denotes the metric of the $3 D$ Minkowski spacetime. Using the triad of components $e_{(a)}^{\mu}$ composing the orthonormal frame, we can obtain the curved spacetime dependent matrices $\bar{\sigma}^{\mu}$ in terms of the constant matrices as follows:

$\bar{\sigma}^{\mu}=e_{(a)}^{\mu} \bar{\sigma}^{(a)}$.

The Greek indices $(\mu, \nu)$ represent the external (global) spacetime indices, and the Latin indices $(a, b)$ denote the internal (local) indices. Hence, the metric tensor is given by

$g_{\mu \nu}=e_{\mu}^{(a)} e_{\nu}^{(b)} \eta_{(a b)}$.

Ultimately, as formulated in [40], the UDE of a fermion ( $\operatorname{spin}-\frac{1}{2}$ ) with mass $m_{s}$ and wave function (spinor) $\Psi_{s}$ in $3 D$ curved spacetime is given by

$i \bar{\sigma}^{\mu}\left[\partial_{\mu}-\Gamma_{\mu}\right] \Psi_{s}=\frac{m_{s}}{\hbar} \Psi_{s}$,

where $\Gamma_{\mu}$ is the spinorial affine connection:

$\Gamma_{\mu}=H_{\lambda v \mu} s^{\lambda v}$.

The rank-3 tensor $H_{\lambda v \mu}$ and the spin operator $s^{\lambda v}$ are, respectively, given by

$H_{\lambda \nu \mu}=\frac{1}{4} g_{\lambda \alpha}\left[e_{\nu, \mu}^{(i)} e_{(i)}^{\alpha}-\Gamma_{\nu \mu}^{\alpha}\right]$,

$s^{\lambda \nu}=\frac{1}{2}\left[\sigma^{\lambda}, \sigma^{\nu}\right]$,

where $\Gamma_{v \mu}^{\alpha}$ is the Christoffel symbol. The following is a possible triad for the metric (1):

$e_{\mu}^{(i)}=\left(\begin{array}{ccc}-\sqrt{f} & 0 & -f^{\theta} r \\ 0 & -\frac{1}{\sqrt{f}} & 0 \\ 0 & 0 & -r\end{array}\right)$,

which yields the following constant matrices:

$\bar{\sigma}^{\mu}=\left(-\frac{\sigma^{(3)}}{\sqrt{f}},-i \sqrt{f} \sigma^{(1)}, \frac{r f^{\theta} \sigma^{(3)}-i \sqrt{f} \sigma^{(2)}}{\sqrt{f} r}\right)$.

Hence from Eq. (48), we compute the non-zero $H_{\lambda v \mu}$ components as

$$
\begin{aligned}
& H_{\theta r \theta}=-H_{r \theta \theta}=\frac{1}{4} r, \\
& H_{t \theta r}=-H_{\theta t r}=\frac{1}{8} r^{2}\left(f^{\theta}\right)^{\prime}, \\
& H_{t r \theta}=H_{\theta r t}=-H_{r \theta t}=-H_{r t \theta}=\frac{1}{4} r f^{\theta}+\frac{1}{8} r^{2}\left(f^{\theta}\right)^{\prime}, \\
& H_{r t t}=-H_{t r t}=\frac{1}{8} f^{\prime}-\frac{1}{4} r\left(f^{\theta}\right)^{2}-\frac{1}{4} r^{2} f^{\theta}\left(f^{\theta}\right)^{\prime} .
\end{aligned}
$$


Subsequently, the spinorial affine connections (47) are evaluated as

$$
\begin{aligned}
\Gamma_{t}= & \frac{1}{4}\left[f^{\prime}-r^{2} f^{\theta}\left(f^{\theta}\right)^{\prime}\right] \sigma^{(2)} \\
& +i \frac{\sqrt{f}}{4}\left[2 f^{\theta}+r\left(f^{\theta}\right)^{\prime}\right] \sigma^{(3)}, \\
\Gamma_{r}= & \frac{r\left(f^{\theta}\right)^{\prime}}{4 \sqrt{f}} \sigma^{(1)}, \\
\Gamma_{\theta}= & -\frac{r^{2}\left(f^{\theta}\right)^{\prime}}{4} \sigma^{(2)}+i \frac{\sqrt{f}}{2} \sigma^{(3)} .
\end{aligned}
$$

The UDE (46) can then be explicitly expressed as

$$
\begin{gathered}
-i \frac{\sigma^{(3)}}{\sqrt{f}} \partial_{t} \Psi_{s}+\sqrt{f} \sigma^{(1)} \partial_{r} \Psi_{s}+\left(\frac{1}{r} \sigma^{(2)}+i \frac{f^{\theta}}{\sqrt{f}} \sigma^{(3)}\right) \partial_{\theta} \Psi_{s} \\
+\left(\frac{f^{\prime}}{4 \sqrt{f}}+\frac{\sqrt{f}}{2 r}\right) \sigma^{(1)} \Psi_{s}-\frac{\left(f^{\theta}\right)^{\prime} r}{4} I_{2 X 2} \Psi_{s}=\frac{m_{s}}{\hbar} \Psi_{s},
\end{gathered}
$$

where $I_{2 \times 2}$ is the $2 \times 2$ unitary matrix. Equation (56) matches with the result of [43]. Now, using the following ansätz for the spinor:

$$
\Psi_{s}=\left\{\begin{array}{l}
\widetilde{A}(t, r, \theta) \exp \left[\frac{i}{\hbar} I(t, r, \theta)\right] \\
\widetilde{B}(t, r, \theta) \exp \left[\frac{i}{\hbar} I(t, r, \theta)\right]
\end{array}\right\}
$$

(recall that $I(t, r, \theta)$ represents the action), we obtain a pair of coupled equations (to the leading order in $\hbar$ ):

$$
\begin{aligned}
& \frac{\widetilde{A}}{\sqrt{f}} \partial_{t} I+i \sqrt{f} \widetilde{B} \partial_{r} I+\left(\frac{\widetilde{B}}{r}-\frac{\widetilde{A} f^{\theta}}{\sqrt{f}}\right) \partial_{\theta} I=m_{s} \widetilde{A}, \\
& -\frac{\widetilde{B}}{\sqrt{f}} \partial_{t} I+i \sqrt{f} \widetilde{A} \partial_{r} I+\left(\frac{\widetilde{B} f^{\theta}}{\sqrt{f}}-\frac{\widetilde{A}}{r}\right) \partial_{\theta} I=m_{s} \widetilde{B} .
\end{aligned}
$$

Equations (58) and (59) have non-trivial solutions for $\widetilde{A}$ and $\widetilde{B}$ provided that the determinants of the coefficient matrices vanish. Hence, we have

$$
\frac{1}{f}\left(\partial_{t} I-f^{\theta} \partial_{\theta} I\right)^{2}-f\left(\partial_{r} I\right)^{2}-\frac{1}{r^{2}}\left(\partial_{\theta} I\right)^{2}-m_{s}^{2}=0 .
$$

By the same process as the previous section, we insert ansätz (29) into Eq. (60) and obtain the following integral solution for $W(r)$ :

$$
W_{ \pm}(r)= \pm \int \frac{\sqrt{\left(E+L f^{\theta}\right)^{2}-f\left[\left(\frac{L}{r}\right)^{2}+m_{s}^{2}\right]}}{f} .
$$

The above equation is structurally very similar to Eq. (30). Naturally, Eq. (61) reduces to Eq. (32) near the event horizon, and consequently, yields the tunneling rate computed by Eq. (38). We remark that, similar to the scalar radiation, the temperature of fermions radiated from the event horizon of a $3 D$ RSHBH is the standard $T_{\mathrm{H}}(21)$.

\section{Conclusion}

In this paper, we investigated the HR of scalar and Dirac particles diverging from the event horizon of a $3 D$ RSHBH. For this purpose, we separated the KGE and UDE on the $3 D$ RSHBH geometry using particular ans ätze for the wave functions Eqs. (27) and (57), respectively. We calculated the quantum tunneling rates of the scalar particles and fermions using the first-order WKB approximation, thereby demonstrating the effect of scalar hair on the tunneling rate of a rotating BTZ BH. Remarkably, both tunneling rates were identical regardless of particle type. After substituting the tunneling rate into the Boltzmann formula, Eq. (39), we recovered the original $T_{\mathrm{H}}(21)$ of the $3 D$ RSHBH.

Finally, whether the results are modified in other hairy BHs, such as BHs with Abelian Higgs hair [44], is an interesting question and will be investigated in our future work.

Open Access This article is distributed under the terms of the Creative Commons Attribution 4.0 International License (http://creativecomm ons.org/licenses/by/4.0/), which permits unrestricted use, distribution, and reproduction in any medium, provided you give appropriate credit to the original author(s) and the source, provide a link to the Creative Commons license, and indicate if changes were made. Funded by $\mathrm{SCOAP}^{3}$.

\section{References}

1. C.W. Misner, K.S. Thorne, J.A. Wheeler, Gravitation (W. H. Freeman, San Francisco, 1973)

2. S.W. Hawking, Commun. Math. Phys. 43, 99 (1975); Erratum 46, 206 (1976)

3. R.M. Wald, Living Rev. Relat. 4, 6 (2001)

4. L. Vanzo, G. Acquaviva, R.D. Criscienzo, Class. Quantum Grav. 28, 183001 (2011)

5. C. Rovelli, Quantum Gravity (Cambridge University Press, Cambridge, 2007)

6. S.W. Hawking, Phys. Rev. D 72, 084013 (2005)

7. A. Aguirre, B. Foster, Z. Merali, It From Bit or Bit From It? On Physics and Information (Springer, New York, 2015)

8. P. Kraus, F. Wilczek, Mod. Phys. Lett. A 09, 3713 (1994)

9. M.K. Parikh, F. Wilczek, Phys. Rev. Lett. 85, 5042 (2000)

10. S. Iso, H. Umetsu, F. Wilczek, Phys. Rev. D 74, 044017 (2006)

11. I. Sakalli, A. Ovgun, EPL 110, 10008 (2015)

12. S. Bhattacharya, A. Lahiri, Phys. Rev. Lett. 99, 201101 (2007)

13. C.A.R. Herdeiro, E. Radu, Phys. Rev. Lett. 112, 221101 (2014)

14. C. Herdeiro, E. Radu, Class. Quantum Grav. 32, 144001 (2015)

15. C.A.R. Herdeiro, E. Radu, Int. J. Mod. Phys. D 24, 1542014 (2015)

16. T. Torii, K. Maeda, M. Narita, Phys. Rev. D 64, 044007 (2001)

17. D. Sudarsky, J.A. González, Phys. Rev. D 67, 024038 (2003)

18. T. Hertog, K. Maeda, JHEP 07, 051 (2004)

19. W. Xu, L. Zhao, Phys. Rev. D 87, 124008 (2013)

20. B. Zuckerman, M.A. Malkan, M.J. Thomas, The Origin and Evolution of the Universe (Jones \& Bartlett Learning, London, 1996)

21. S. Deser, R. Jackiw, G. 't Hooft, Ann. Phys. 152, 220 (1984)

22. S. Deser, R. Jackiw, Commun. Math. Phys. 118, 495 (1988)

23. G. 't Hooft, Commun. Math. Phys. 117, 685 (1988)

24. E. Witten, Nucl. Phys. B 311, 46 (1988) 
25. E. Witten, Nucl. Phys. B 323, 113 (1989)

26. S. Carlip, J. Korean Phys. Soc. 28, 447 (1995)

27. L. Zhao, W. Xu, B. Zhu, Commun. Theor. Phys. 61, 475 (2014)

28. D.C. Zou, Y. Liu, B. Wang, W. Xu, Phys. Rev. D 90, 104035 (2014)

29. A. Belhaj, M. Chabab, H. El Moumni, K. Masmar, M.B. Sedra, Int. J. Geom. Methods Mod. Phys. 12, 1550017 (2015)

30. J. Naji, H. Saadat, Adv. High Energy Phys. 2014, 720713 (2014)

31. M. Banãdos, C. Teitelboim, J. Zanelli, Phys. Rev. Lett. 69, 1849 (1992)

32. M. Banãdos, M. Henneaux, C. Teitelboim, J. Zanelli, Phys. Rev. D 48, 1506 (1993)

33. S. Carlip, Class. Quantum Grav. 22, R85 (2005)

34. B.M. Karnakov, V.P. Krainov, WKB Approximation in Atomic Physics (Springer, New York, 2013)

35. C. Smorynski, History of Mathematics: A Supplement (Springer, New York, 2008)
36. P. Kraus, E. Keski-Vakkuri, Nucl. Phys. B 491, 249 (1997)

37. R.M. Wald, General Relativity (The University of Chicago Press, Chicago, London, 1984)

38. F. Darabi, K. Atazadeh, A. Rezaei-Aghdam, Eur. Phys. J. C 74, $2967(2014)$

39. K. Srinivasan, T. Padmanabhan, Phys. Rev. D 60, 024007 (1999)

40. Y. Sucu, N. Unal, J. Math. Phys. 48, 052503 (2007)

41. J.P.M. Pitelli, P.S. Letelier, J. Math. Phys. 48, 092501 (2007)

42. O. Unver, O. Gurtug, Phys. Rev. D 82, 084016 (2010)

43. F. Belgiorno, S.L. Cacciatori, F.D. Piazza, O.F. Piattella, J. Phys. A 44, $025202(2011)$

44. A. Achhcarro, R. Gregory, K. Kuijkent, Phys. Rev. D 52, 5729 (1995) 